\title{
A constructive approach to regularity of Lagrangian trajectories for incompressible Euler flow in a bounded domain
}

\author{
Nicolas Besse ${ }^{\dagger}$, Uriel Frisch ${ }^{\ddagger}$ \\ September 18, 2018
}

\begin{abstract}
The 3D incompressible Euler equation is an important research topic in the mathematical study of fluid dynamics. Not only is the global regularity for smooth initial data an open issue, but the behaviour may also depend on the presence or absence of boundaries.

For a good understanding, it is crucial to carry out, besides mathematical studies, highaccuracy and well-resolved numerical exploration. Such studies can be very demanding in computational resources, but recently it has been shown that very substantial gains can be achieved first, by using Cauchy's Lagrangian formulation of the Euler equations and second, by taking advantages of analyticity results of the Lagrangian trajectories for flows whose initial vorticity is Hölder-continuous. The latter has been known for about twenty years (Serfati, J. Math. Pures Appl. 74: 95-104, 1995), but the combination of the two, which makes use of recursion relations among time-Taylor coefficients to obtain constructively the time-Taylor series of the Lagrangian map, has been achieved only recently (Frisch and Zheligovsky, Commun. Math. Phys. 326: 499-505, 2014; Podvigina et al., J. Comput. Phys. 306: 320-342, 2016 and references therein).

Here we extend this methodology to incompressible Euler flow in an impermeable bounded domain whose boundary may be either analytic or have a regularity between indefinite differentiability and analyticity. Non-constructive regularity results for these cases have already been obtained by Glass et al. (Ann. Scient. Éc. Norm. Sup. $4^{e}$ série 45: 1-51, 2012). Using the invariance of the boundary under the Lagrangian flow, we establish novel recursion relations that include contributions from the boundary. This leads to a constructive proof of time-analyticity of the Lagrangian trajectories with analytic boundaries, which can then be used subsequently for the design of a very high-order Cauchy-Lagrangian method.
\end{abstract}

Keywords: 3D incompressible Euler equation; ideal fluids in bounded domains; Cauchy Lagrangian formulation; smoothness of particle trajectories; time-Taylor series; recursion relations; ultradifferentiable function spaces; Hölder spaces.

AMS: to be completed

\section{Introduction}

The Cauchy problem for the 3D incompressible Euler equation is known to be well posed in time when the initial velocity is in Hölder or Sobolev spaces with suitable indexes of regularity. This was established in the seminal work of Lichtenstein [47, 48] and Gyunter [31, 32] for the case of the whole space and, of Ebin and Marsden 22] for the case of bounded domains. In other words, limited initial spatial smoothness is preserved in time, at least for a finite duration. Here is a typical

\footnotetext{
${ }^{\dagger}$ Laboratoire J.-L. Lagrange, UCA, OCA, CNRS, CS 34229, 06304 Nice Cedex 4 France (Nicolas.Besse@oca.eu)
}

${ }^{\ddagger}$ Laboratoire J.-L. Lagrange, UCA, OCA, CNRS, CS 34229, 06304 Nice Cedex 4 France (uriel@oca.eu) 
result: Suppose the initial velocity $v_{0} \in \mathscr{C}^{m, \gamma}$, with $m \in \mathbb{N}-\{0\}$ and $0<\gamma<1$ (see, e.g., [1] for the definition of such Hölder spaces). It needs to be just a little better than $\mathscr{C}^{1}$ for the existence of a unique solution $v(t, \cdot) \in \mathscr{C}^{m, \gamma}$ for $|t|<T\left(\left\|v_{0}\right\|_{\mathscr{C}} m, \gamma\right)$. Moreover, the solution $v(t, \cdot)$ has exactly the same spatial regularity as the initial velocity $v_{0}$, i.e. if $v(t, \cdot) \in \mathscr{C}^{n, \eta}$ with $n>m$ and $\eta>\gamma$ for some $t>0$, then $v_{0} \in \mathscr{C}^{n, \eta}$. Nevertheless, the issue of loss of regularity after a finite time (blow-up) of solutions for the 3D incompressible Euler equation still remains an open problem.

In Eulerian coordinates, lack of regularity in space also translates into a lack of regularity in time. For instance, in Ref. [10] are constructed periodic weak solutions of the 3D incompressible Euler equation, which dissipate the total energy and which belong to the Hölder space of exponent $\gamma<1 / 5(m=0)$, in both space and time. By contrast in Lagrangian coordinates, where one is focusing on particle trajectories, an initial velocity field with limited smoothness (typically in Hölder classes with suitable indexes of regularity) launches characteristic curves whose temporal smoothness (typically in some indefinitely differentiable function classes) widely exceeds the limited spatial smoothness. These are the kinds of typical statements that we will prove here.

Actually, it was already pointed out in Ref. [22] that the Lagrangian structure can be nicer than the Eulerian one. For this, it is convenient to use the geometric interpretation [2] of the Euler equations in Lagrangian coordinates to show that "no loss of derivative occurs". From a technical point of view, the simplest way of proving a "no loss of derivative" result is to start from Cauchy's Lagrangian formulation of the Euler equation [13, 26]. This leads to a very simple and constructive proof of time-analyticity of the Lagrangian trajectories [25, 67]. Another recent related work [17], uses a Lagrangian formulation of the Euler equation to revisit some known results concerning the decay of the Eulerian analyticity radius in space variables [6, 4, 46, 42, 43, 44, 68] and to show the persistence of the spatial Lagrangian analyticity radius.

It is of interest to recall how the issue of Lagrangian regularity was tackled so far. The temporal smoothness of Lagrangian particle paths, was first studied by Chemin ([14]; see also [15]) in Eulerian coordinates, using a paradifferential approach. He showed that Lagrangian trajectories are $\mathscr{C}^{\infty}$ regular when initial conditions are in the Hölder classes $\mathscr{C}^{m, \gamma}(m \in \mathbb{N}-\{0\})$. Chemin's proof relies on the estimate of the repeated action of the material derivative $\partial_{t}+v \cdot \nabla$ on the pressure, which is expressed via a nonlinear pseudodifferential operator. In Refs. 62, 63, 64, Serfati extended Chemin's results to the case of temporal analyticity and spatial Hölder regularity $\left(\mathscr{C}^{m, \gamma}\right.$ with $m \in$ $\mathbb{N}-\{0\}$ ). In the spirit of Refs., [9] for an Euclidean space, or [22] for Riemannian manifolds, Serfati suggested to attack the problem as an abstract ordinary differential equation (ODE) in a complex Banach space, holomorphic in time. The right-hand side of the ODE is obtained by using the BiotSavart-type singular integral representation of the pressure, extended to an analytic functional on a complex Banach space. This requires a careful analytic extension of the Newtonian potential singularity on a sector of the $n$-dimensional complex plane. Serfati proved Banach-space analyticity of such a functional, and thus its Lipschitz property, by using the Cauchy integral formula and showing boundedness of the functional in a complex Banach space. More recently, Shnirelman 65] obtained another analytic abstract ODE formulation in complex Banach spaces, which makes use of Arnold's geometric interpretation of the Euler equation [2]. The latter formulation uses the Biot-Savart-type singular integral representation of the stream-function, and takes advantage of Cauchy's vorticity formula in Lagrangian coordinates [13] to obtain a divergence-free representation of the velocity field and discard the pressure. Serfati [64] and Shnirelman [65] constructed a time-analytic Lagrangian solution to the ODE by using Picard successive approximations and a Banach fixed-point argument, like in the proof of the Cauchy-Lipschitz-Picard theorem. Another temporal analyticity result, which follows the spirit of Chemin's approach, is found in Ref. [27. Yet another approach, applicable to a class of hydrodynamic-like equations with nonlinear and nonlocal (collective) interactions uses singular integral operators (SIO); its proof makes crucial use of the Faà di Bruno formula [23, 33, 18, 38] to deal with successive time derivatives of the composition of 
the SIO kernel function with the flow map [16].

All the previous regularity results were established for fluids without boundaries: either filling the whole space or with periodic boundary conditions. In the presence of a boundary, using Eulerian coordinates, Kato [35] showed $\mathscr{C}^{\infty}$-regularity in time of the Lagrangian trajectories for Hölderian classical solutions of the $n$-dimensional Euler equation in a smooth bounded domain with a boundary of $\mathscr{C}^{\infty}$-regularity. Kato's approach involved commutators of the material derivative and of the differential operators arising from the Helmholtz-Hodge decomposition of the vector-valued Euler equation. For these commutators, Kato obtained key estimates in both the interior of the domain and - even more important - its boundary. Of course these commutator estimates involve a representation of the boundary. Specifically the author used successive derivatives of the smooth function measuring the distance between an interior point to the boundary. Nonlocal features, due to the pressure term, were tackled by using classical elliptic regularity estimates. Refinements of Kato's estimates have recently led to a proof of analyticity in time of the Lagrangian trajectories for Hölderian classical solution of the 3D Euler equation in the presence of an analytic boundary 30].

The various methods we have briefly reviewed above are not constructive in the following sense: they cannot be used to construct a high-order numerical scheme for integrating the Euler equation in either Eulerian or Lagrangian coordinates. A constructive approach follows from Cauchy's [13] Lagrangian formulation. As shown in Refs. [13] this formulation leads to simple recursion relations among the time-Taylor coefficients of the Lagrangian flow application mapping initial fluid particle positions to current ones. It is then possible not only to prove time-analyticity, but also to construct efficiently very accurate solutions to the Euler equation [56]. Here, we note that the terminology "recursion relation" is borrowed from the perturbation theory, as used in cosmology for over twenty years (see, e.g., [7] and references therein).

It is our main goal to show that such a constructive method can be extended to Euler flow with a boundary. The outline of the paper is as follows. In Sec. 2, we introduce our notation and define our functional framework (Sec. 2.1). Then, in Sec. 2.2 we state our main result, namely Theorem 2, a corollary of which is time-analyticity. In Sec. 3, we present the proof of Theorem 2 in two steps. First, in Sec. 3.1 we construct the time-Taylor coefficients by solving a sequence of non-homogeneous Dirichlet and Neumann boundary value problems. The boundary conditions on these are given by boundary recursion relations stemming from the preservation of the boundary by the Euler flow (impenetrability). Then, in Sec. 3.2, we deal with convergence issues and obtain bounds (e.g. in Hölder spaces) for time-Taylor coefficients, from which the proof of Theorem 2 follows. Sec. 4 is devoted to concluding remarks and to discussing open problems.

\section{Smoothness of trajectories for an incompressible ideal fluid in a bounded domain}

In this section, we first define some notation and functional spaces. Then, we state our main theorem. Actually, our functional framework consists of different classes of indefinitely differentiable functions, that encompass analytic, quasi-analytic, and non-quasi-analytic classes. We will perform our study in a general ultradifferentiable class [8, 60, 50, 37] that we call the "log-superlinear Faà-di-Bruno" class.

\subsection{Notation and functional framework}

Let $N^{*}$ be the positive numbers of the set of natural integers $\mathbb{N}$. For a multi-index $\alpha=\left(\alpha_{1}, \ldots, \alpha_{d}\right) \in$ $\mathbb{N}^{d}$ and $x=\left(x_{1}, \ldots, x_{d}\right) \in \mathbb{R}^{d}$, we use the standard notation $\alpha !:=\alpha_{1} ! \ldots \alpha_{d} !, \quad|\alpha|:=\alpha_{1}+\ldots+\alpha_{d}$ 
and $x^{\alpha}:=x_{1}^{\alpha_{1}} \ldots x_{d}^{\alpha_{d}}$. We use $\partial_{i}:=\partial / \partial x_{i}, \partial^{\alpha}:=\partial_{1}^{\alpha_{1}} \ldots \partial_{d}^{\alpha_{d}}$, and we write $D^{s} f$ for the $s$-th Fréchet (functional) derivative of $f$.

We start with some definitions for ultradifferentiable classes, a generalization of Gevrey classes [52, 50]. Let $U$ be a domain in $\mathbb{R}^{d}$ and let $\mathfrak{B}$ be a Banach space endowed with the norm $\|\cdot\|_{\mathfrak{B}}$. Let $f: U \longrightarrow \mathfrak{B}$ be an infinitely Fréchet-differentiable map. Then the $s$-th Fréchet derivative of $f$, $D^{s} f: U \longrightarrow \mathcal{L}^{s}(U \times \ldots \times U, \mathfrak{B})$, is an element of $\mathcal{L}^{s}(U \times \ldots \times U, \mathfrak{B})$, the set of $s$-linear bounded or continuous maps from $U \times \ldots \times U$ to $\mathfrak{B}$. The space $\mathcal{L}^{\mathcal{s}}(U \times \ldots \times U, \mathfrak{B})$ is endowed with the standard operator norm $\|\cdot\|\|:=\| \cdot \|_{\mathcal{L}^{s}(U \times \ldots \times U, \mathfrak{B})}$.

Let $\mathcal{M}:=\left\{\mathcal{M}_{s}\right\}_{s \geq 0}$ be a sequence of positive numbers. The ultradifferentiable class $\mathcal{C}\{\mathcal{M}\}(U ; \mathfrak{B})$ is defined as the set of infinitely Fréchet-differentiable functions $f: U \longrightarrow \mathfrak{B}$ such that for any compact set $K \subset U$ there exist constants (depending on $f$ ) $R_{f}, C_{f}$ such that for all $s \in \mathbb{N}$,

$$
\sup _{x \in K}\left\|\left|D^{s} f(x)\right|\right\| \leq C_{f} R_{f}^{-s} \mathcal{M}_{s} .
$$

The class $\mathcal{C}\{\mathcal{M}\}$ is invariant under multiplication by a constant, i.e. $\mathcal{C}\{\lambda \mathcal{M}\}(U ; \mathfrak{B})=\mathcal{C}\{\mathcal{M}\}(U ; \mathfrak{B})$ for $\lambda>0$. There exists a fairly large set of ultradifferentiables functions [52, 60, 37]. Here we choose a class that we call the "log-superlinear Faà-di-Bruno" (LSL-FdB in short) class. It involves a sequence of weights $M:=\left\{\mathcal{M}_{s} / s !\right\}_{s \geq 0}$ (and $M_{0}=\mathcal{M}_{0}$ ), in terms of which we have the following

Definition 1 The log-superlinear Faà-di-Bruno class is the set of functions satisfying (1) with the following restriction on the weights:

i) differentiation stability:

$$
\exists C_{\mathrm{d}}>0: \quad M_{k+1} \leq C_{\mathrm{d}}^{k} M_{k}, \quad \forall k \in \mathbb{N} .
$$

ii) log-superlinearity:

$$
M_{k} M_{\ell} \leq M_{0} M_{k+\ell}, \quad \forall k, \ell \in \mathbb{N}
$$

iii) (FdB)-stability:

$$
\exists 0<C_{\mathrm{FdB}} \leq 1 \mid \forall \alpha_{i} \in \mathbb{N}^{*}, \alpha_{1}+\ldots+\alpha_{\ell}=k: \quad M_{\ell} M_{\alpha_{1}} \ldots M_{\alpha_{\ell}} \leq C_{\mathrm{FdB}}^{k} M_{k} .
$$

A few remarks are now in order.

Remark 1 Using the Leibniz differentiation rules, log-superlinearity implies that the class $\mathcal{C}\{\mathcal{M}\}(U ; \mathfrak{B})$ is an algebra with respect to pointwise multiplication. Using the Faà di Bruno formula [23, [33, 18, 38], (FdB)-stability implies stability under composition in the class $\mathcal{C}\{\mathcal{M}\}(U ; \mathfrak{B})$ (see the proof of Proposition 3.1 of Ref. [57]). Finally, the differentiability stability property implies closure under differentiation in $\mathcal{C}\{\mathcal{M}\}(U ; \mathfrak{B})[60$, 50, 377].

Remark 2 Let us observe that this class is slightly larger than the log-convex class used in Ref. [30], in which the weights satisfy the following equivalent properties:

1. $k \longmapsto \log M_{k}$ is convex.

2. $M_{k}^{2} \leq M_{k-1} M_{k+1}, \forall k \in \mathbb{N}$.

3. The sequence $\left\{M_{s+1} / M_{s}\right\}_{s \geq 0}$ is nondecreasing. 
It is straightforward to show that log-convexity implies log-superlinearity (3) but the converse does not hold. Moreover, it is shown in Lemma 2.2 of Ref. [57] (see also [58, 39]) that log-convexity implies (FdB)-stability with an (FdB)-stability constant $C_{\mathrm{FdB}}=\max \left\{1, M_{1}\right\}$. Since the class $\mathcal{C}\{\mathcal{M}\}$ is invariant under multiplication by a constant, we can normalize the sequence $M=\left\{M_{k}\right\}_{k \geq 0}$ by an arbitrary positive constant. Therefore, dividing $M$ by $M_{1}$ implies $C_{\mathrm{FdB}}=\max \left\{1, M_{1}\right\}=1$. In other words log-convexity implies ( $F d B)$-stability (4). Let us note that usually in the literature [8, 60, 50, 37, 66, 39, 40, 41, 57, 58], ultradifferentiable classes assume log-convexity in order to achieve stability under composition. We refer the reader to [57, 58] and references therein for a discussion of the role of log-convexity in achieving stability under composition.

Remark 3 Some well-known classes of functions belong to the LSL-FdB class. The first one is the analytic functions class which corresponds to $M_{k}=1$ (e.g. [52, 12]). The second one is the Gevrey class (see, e.g., [52, 50] and references therein) which corresponds to $M_{k}=(k !)^{r}$ with $r>0$. In the usual technical sense, the former is also quasi-analytic and the latter is not [61, 8, 34, 66, 39, 40] (see also 441]). Recall that ultradifferentiable classes are divided into quasianalytic and non-quasi-analytic classes, depending on whether the map to infinite Taylor expansions is injective on the class or not (see, e.g., [66, 39, 40]). Denjoy [20] and Carleman [11] have given some criteria for distinguishing quasi-analytic classes from non-quasi-analytic ones. Typically, the Denjoy-Carleman theorem [20, 11] states that $\mathcal{C}\{\mathcal{M}\}(U ; \mathfrak{B})$ lacks quasi-analyticity if and only if $\sum_{k>0} M_{k} / M_{k+1}<\infty$ (see, e.g., [61, 34, 37, 66, 39, 40] for contemporary proofs). We refer the reader to [8, 60, 37, 34, 61, 66, 39, 40, 41] and references therein for establishing various equivalent Denjoy-Carleman-type criteria.

The ultradifferentiable framework will be used here for studying the regularity in time. It remains to choose a functional space to measure the regularity in space of the initial data, i.e. the regularity with respect to the Lagrangian spatial variable. Two requirements are needed for this functional space. First, as we shall see, it must be an algebra with respect to the pointwise multiplication. Second, it must be compatible with local-in-time well-posedness results of the 3D incompressible Euler equation (see Theorem 1 below). For convenience, we choose Hölder spaces, but our proof can be extended, for instance, to Sobolev and Besov spaces (with suitable indexes of regularity), or ultradifferentiable spaces (e.g., analytic functions). We recall the definition of the Hölder spaces $\mathscr{C}^{m, \gamma}$ used here and an associated basic property. Let $\Omega$ be a bounded domain in $\mathbb{R}^{d}$ with $d \geq 1$. Let $\mathscr{C}^{m}(\bar{\Omega})$ be the space of $m$ times continuously differential functions on the closure $\bar{\Omega}$, equipped with the norm

$$
\|f\|_{m} \equiv\|f\|_{\mathscr{C} m}(\bar{\Omega}):=\max _{0 \leq|\alpha| \leq m} \sup _{x \in \bar{\Omega}}\left|\partial^{\alpha} f(x)\right| .
$$

For $0<\gamma<1$ and $m \in \mathbb{N}$, the Hölder space $\mathscr{C}^{m, \gamma}(\bar{\Omega})$ is defined as the subspace of $\mathscr{C}^{m}(\bar{\Omega})$ consisting of those functions $f$ for which, for $0<|\alpha| \leq m, \partial^{\alpha} f$ satisfies in $\Omega$ a Hölder condition of exponent $\gamma$. The space $\mathscr{C}^{m, \gamma}(\bar{\Omega})$ is endowed with the norm

$$
\|f\|_{m, \gamma} \equiv\|f\|_{\mathscr{C} m, \gamma(\bar{\Omega})}:=\|f\|_{m}+\max _{0 \leq|\alpha| \leq m} \sup _{\substack{x, y \in \bar{\Omega} \\ x \neq y}} \frac{\left|\partial^{\alpha} f(x)-\partial^{\alpha} f(y)\right|}{|x-y|^{\gamma}} .
$$

The space $\mathscr{C}^{m, \gamma}(\bar{\Omega})$ is an algebra with respect to the pointwise multiplication (see, e.g., Chapter 4 of [51], or Chapter 4 of [28]), i.e. there exists a constant $C_{a}:=C_{a}(m)$, which depends on $m$, but is independent of $\gamma$, such that

$$
\|f g\|_{m, \gamma} \leq C_{a}\|f\|_{m, \gamma}\|g\|_{m, \gamma}, \quad \forall f, g \in \mathscr{C}^{m, \gamma}(\bar{\Omega}) .
$$


Finally we introduce the notation $\mathcal{D}^{\prime}(\Omega)$ for the space of (Schwartz) distribution on $\Omega$. The space $\mathcal{D}^{\prime}(\Omega)$ is defined in a standard way as the topological dual of the space of indefinitely differentiable functions with compact support on $\Omega$.

\subsection{Main theorem}

Before tackling issues of Lagrangian time analyticity and variants thereof, we state a key result of Kato about the initial-value Euler problem in the presence of a boundary [35]. Kato's result applies in particular to an incompressible ideal fluid filling a bounded simply connected regular domain $\Omega \subset \mathbb{R}^{3}$, as we shall assume throughout this paper. The relevant equations (so far in Eulerian coordinates) are:

$$
\begin{aligned}
& \left.\partial_{t} v+(v \cdot \nabla) v+\nabla p=0, \quad x \in \Omega, \quad t \in\right]-T, T[ \\
& \nabla \cdot u=0, \quad x \in \Omega, \quad t \in]-T, T[ \\
& v_{\mid t=0}=v_{0}, \quad x \in \Omega \\
& v \cdot \nu=0, \quad x \in \partial \Omega, \quad t \in]-T, T[.
\end{aligned}
$$

Here $v$ is the velocity, $p$ the pressure and $\nu$ the outer normal at the impermeable boundary $\partial \Omega$.

We then have the following

Theorem 1 (Kato [35]). Suppose that $(v, p)$ is a solution of $\left([6)-(9)\right.$, where $v \in \mathscr{C}(] 0, T\left[; \mathscr{C}^{1, \gamma}(\bar{\Omega})\right)$ with $\gamma \in(0,1)$, and $p \in \mathcal{D}^{\prime}(] 0, T[\times \Omega)$. Then we have, for all $s \in \mathbb{N}^{*}$,

$$
\left(\partial_{t}+v \cdot \nabla\right)^{s} v \in \mathscr{C}(] 0, T\left[\mathscr{C}^{1, \gamma}(\bar{\Omega})\right), \quad \nabla\left(\partial_{t}+v \cdot \nabla\right)^{s-1} p \in \mathscr{C}(] 0, T\left[; \mathscr{C}^{0, \gamma}(\bar{\Omega})\right),
$$

and

$$
\left.\left\|\left(\partial_{t}+v \cdot \nabla\right)^{s} v(t)\right\|_{1, \gamma}+\left\|\nabla\left(\partial_{t}+v \cdot \nabla\right)^{s-1} p(t)\right\|_{0, \gamma} \leq C_{s}\|v(t)\|_{1, \gamma}^{s+1}, \quad \forall t \in\right] 0, T[,
$$

where $C_{s}$ are constants depending on $\gamma, s$ and $\Omega$.

Remark 4 We choose here the Hölder space $\mathscr{C}^{1, \gamma}(\bar{\Omega})$ but, of course, Kato's theorem also holds for Hölder spaces $\mathscr{C}^{m, \gamma}(\bar{\Omega})$ with $m \geq 1$ (see [35]). Theorem 1 relies on existence, uniqueness and regularity properties of the local-in-time classical solution of the Euler equations (6) -(9) in Hölder spaces (see, e.g., Theorem I of [36]]) with initial condition $v_{0} \in \mathscr{C}^{1, \gamma}(\bar{\Omega})$ and such that $T=C_{*}(\Omega) /\left\|v_{0}\right\|_{\mathscr{C}^{1, \gamma}(\bar{\Omega})}$. Moreover, Theorem 1 can be extended for, say, initial conditions in any Sobolev space $H^{s}(\Omega)$ with $s>5 / 2$ and any inhomogeneous Besov space $B_{p, q}^{s}(\Omega)$, with $1 \leq p, q \leq \infty$ and with $s>3 / p+1$ or $s \geq 3 / p+1$ if $q=1$, so that $B_{p, q}^{s}(\Omega)$ is continuously embedded in the Lipschitz space $\operatorname{Lip}(\Omega)$ (see [21]). Let us recall that it is still not known whether the classical solutions of Theorem 1 remain smooth for all times or "blow-up" in finite time. Let us also point out the recent work by Bardos and Titi [5] showing that the 3D Euler equations are not well-posed in the Hölder spaces $\mathscr{C}^{0, \gamma}(\bar{\Omega})$ for $\gamma \in(0,1)$.

For the statement of our main theorem we need to introduce the Lagrangian map $X$ defined on ]$-T, T[\times \Omega$ by

$$
\partial_{t} X(t, a)=v(t, X(t, a)) \quad \text { and } \quad X(0, a)=a .
$$

The Lagrangian map $X(t, \cdot): \Omega \longrightarrow \Omega$ can been seen as a continuous one-parameter group of volume and orientation preserving diffeomorphisms defined on $\Omega$ [2]. Fields expressed in terms of the Lagrangian label $a$ and time $t$ are said to be given in Lagrangian coordinates. The Lagrangian gradient, with respect to $a$, is denoted $\nabla^{\mathrm{L}}$ and its components $\partial_{i}^{\mathrm{L}}$.

The main result of this paper states roughly that the smoothness of the Lagrangian characteristic curves is only limited by the smoothness of the domain boundary. More precisely, we have 
Theorem 2 Assume that the hypotheses of Theorem 1 hold, and in addition that the boundary $\partial \Omega$ belongs to $\mathcal{C}\{\mathcal{M}\}$, where $\mathcal{M}:=\left\{s ! M_{s}\right\}_{s \geq 0}$, with the sequence $\left\{M_{s}\right\}_{s \geq 0}$ satisfying Definition 1 (log-superlinear Fa⿳亠口-di-Bruno class). Then there exists a time $T=C_{*}(\Omega) /\left\|v_{0}\right\|_{\mathscr{C} 1, \gamma(\bar{\Omega})}$ such that the Lagrangian map $X$ satisfies

$$
X \in \mathcal{C}\{\mathcal{M}\}(]-T, T\left[\mathscr{C}^{1, \gamma}(\bar{\Omega})\right)
$$

From Theorem 2 we infer, by specializing to $\mathcal{M}=\{s !\}_{s \geq 0}$ or, equivalently to $M_{s}=1, \forall s \geq 0$, the following analyticity

Corollary 1 Assume that the hypotheses of Theorem 2 hold. If the boundary $\partial \Omega$ is analytic (resp. Gevrey of order $r>0$ ), then Lagrangian map $X$ is analytic (resp. Gevrey of order $r>0$ ) from ]$-T, T\left[\right.$ to $\mathscr{C}^{1, \gamma}(\bar{\Omega})$. For the analytic case, the Lagrangian map $X$ admits a convergent time-Taylor expansion around time $t=0$.

\section{Proof of Theorem 2}

Here, we give a proof of Theorem 2, divided into two steps. Sec. 3.1 is devoted to a recursive construction of a solution, for the Lagrangian map, as a formal time-Taylor expansion, without specifying any functional setting. The second step (Sec. 3.2) is dedicated to convergence issues of such formal time-expansions within the functional framework defined in Sec. 2.1.

\subsection{Construction of a solution as a formal time series}

Our starting point is not the usual Eulerian formulation of the Euler equations (6) -(9) for incompressible ideal fluid, but a little-known Lagrangian formulation due to Cauchy [13], together with the incompressibility condition and the preservation by the Lagrangian flow of a prescribed rigid boundary $\partial \Omega$, represented by the equation $\mathcal{S}(x)=0\left(\mathcal{S}: \mathbb{R}^{3} \rightarrow \mathbb{R}\right.$ being a map of appropriate regularity). The relevant equations are:

$$
\begin{aligned}
\sum_{k=1}^{3} \nabla^{\mathrm{L}} \dot{X}_{k}(t, a) \times \nabla^{\mathrm{L}} X_{k}(t, a) & =\omega_{0}(a), & & \forall a \in \Omega, \\
\operatorname{det}\left(\nabla^{\mathrm{L}} X(t, a)\right) & =1, & & \forall a \in \Omega, \\
\mathcal{S}(X(t, a)) & =0, & & \forall a \in \partial \Omega .
\end{aligned}
$$

Here, the Lagrangian variable $X$ (resp. a) stands for the current (resp. initial) Lagrangian position of fluid particules. The initial vorticity $\omega_{0}$ is defined as usual, i.e. $\omega_{0}=\nabla^{\mathrm{L}} \times v_{0}$, where $\nabla^{\mathrm{L}}$ denotes the gradient in the Lagrangian variables. The dot denotes the Lagrangian time derivative, while $\nabla^{\mathrm{L}} X$ stands for the Jacobian matrix with entries $\partial_{i}^{\mathrm{L}} X_{j}$. The left-hand-side of (13), which is obviously time-invariant, is usually referred to as the "Cauchy invariants."

Now, we observe that the assumptions of our Theorem 2 are always stronger than those of Kato's Theorem 1, given in Section 2.2. Thus, the initial velocity is at least $\mathscr{C}^{1, \gamma}$ and the boundary $\partial \Omega$ has at least $\mathscr{C}^{\infty}$ regularity, from which follows that the Lagrangian map is $\mathscr{C}^{\infty}$ in time. The recursion relations among time-Taylor coefficients derived below could be obtained from the basic Lagrangian equation (13)-(15) by successive time-differentiations and use of standard relations such as the Faà di Bruno formula for differentiation of compositions. This procedure can be somewhat algebraized by using formal time-Taylor series without worrying about convergence issues. Indeed, as we shall see, the relations we will obtain always involve a finite number of terms. Of course, once we address issues such as analyticity, studying the convergence of such formal series becomes 
important. This will however be postponed to Sec. 3.2, where we will derive various functional estimates.

For the implementation, it is convenient, following Refs. [25, 67], to introduce the displacement field $\xi=\xi(t, a)=X-a$, in terms of which, (13) $-(14)$ become

$$
\begin{aligned}
& \nabla^{\mathrm{L}} \times \dot{\xi}+\sum_{1 \leq k \leq 3} \nabla^{\mathrm{L}} \dot{\xi}_{k} \times \nabla^{\mathrm{L}} \xi_{k}=\omega_{0}, \\
& \nabla^{\mathrm{L}} \cdot \xi+\sum_{1 \leq i<j \leq 3}\left(\partial_{i}^{\mathrm{L}} \xi_{i} \partial_{j}^{\mathrm{L}} \xi_{j}-\partial_{i}^{\mathrm{L}} \xi_{j} \partial_{j}^{\mathrm{L}} \xi_{i}\right)+\operatorname{det}\left(\nabla^{\mathrm{L}} \xi\right)=0 .
\end{aligned}
$$

We now use the formal Taylor series for the displacement

$$
\xi(t, a)=\sum_{s>0} \xi^{(s)}(a) t^{s}
$$

Substituting (18) into (16)-(17) and collecting terms of the same power $s>0$, we obtain, after a symmetrization of the sums

$$
\begin{aligned}
\nabla^{\mathrm{L}} \times \xi^{(s)}= & \omega_{0} \delta_{1 s}-\frac{1}{2} \sum_{\substack{1 \leq k \leq 3 \\
0<m<s}} \frac{2 m-s}{s} \nabla^{\mathrm{L}} \xi_{k}^{(m)} \times \nabla^{\mathrm{L}} \xi_{k}^{(s-m)} \\
\nabla^{\mathrm{L}} \cdot \xi^{(s)}= & \sum_{\substack{1 \leq i<j \leq 3 \\
0<m<s}}\left(\partial_{i}^{\mathrm{L}} \xi_{j}^{(m)} \partial_{j}^{\mathrm{L}} \xi_{i}^{(s-m)}-\partial_{i}^{\mathrm{L}} \xi_{i}^{(m)} \partial_{j}^{\mathrm{L}} \xi_{j}^{(s-m)}\right) \\
& -\frac{1}{6} \sum_{l+m+n=s} \varepsilon_{i j k} \varepsilon_{a b c} \partial_{i}^{\mathrm{L}} \xi_{a}^{(l)} \partial_{j}^{\mathrm{L}} \xi_{b}^{(m)} \partial_{k}^{\mathrm{L}} \xi_{c}^{(n)}
\end{aligned}
$$

Here, $\varepsilon_{i j k}$ stands for the unit antisymmetric tensor and $\delta_{i j}$ is the Kronecker symbol. In (201), repeated indices are implicity summed over.

We observe that (19)-(20), which prescribe the Lagrangian curl and divergence of $\xi^{(s)}$, constitute a Helmholtz-Hodge problem (see, e.g., [24, 29, 3]). This is the point where it is essential to handle the boundary conditions, stemming from (15). Indeed, the 3D Helmholtz-Hodge decomposition of the vector field $\xi^{(s)}$ consists in expressing it as follows:

$$
\xi^{(s)}=\nabla^{\mathrm{L}} \varphi^{(s)}+\nabla^{\mathrm{L}} \times \Phi^{(s)}, \quad \text { in } \bar{\Omega},
$$

where $\varphi^{(s)}\left(\right.$ resp. $\left.\Phi^{(s)}\right)$ is the scalar (resp. vector) potential (see, e.g., [24, 29, 3]). Without loss of generality one can assume the gauge condition

$$
\nabla^{\mathrm{L}} \cdot \Phi^{(s)}=0, \quad \text { in } \bar{\Omega} .
$$

By taking the divergence and the curl of (21) and using (22), it is found that the scalar potential $\varphi^{(s)}$ and the vector potential $\Phi^{(s)}$ satisfy, in the interior of $\Omega$, the following non-homogeneous Poisson equations

$$
\Delta^{\mathrm{L}} \varphi^{(s)}=\nabla^{\mathrm{L}} \cdot \xi^{(s)}, \quad \Delta^{\mathrm{L}} \Phi^{(s)}=-\nabla^{\mathrm{L}} \times \xi^{(s)}, \quad \text { in } \Omega,
$$

where $\Delta^{\mathrm{L}}$ is the Lagrangian Laplacian. An important observation is that the boundary traces of the scalar potential $\varphi^{(s)}$ and of the vector potential $\Phi^{(s)}$ on $\partial \Omega$ are obtainable from the flow-invariance of the boundary, expressed by the constraint (15). Indeed, for a given $a \in \partial \Omega$, let us define

$$
f(t)=\mathcal{S}(X(t, a))
$$


which has to vanish, since $X(t, a)$ remains on $\partial \Omega$. We now expand (24) in a (possibly formal) time-Taylor series,

$$
f(t)=\sum_{s \geq 0} f^{(s)}(0) \frac{t^{s}}{s !}, \quad \forall a \in \partial \Omega,
$$

where the $s$-th derivative $f^{(s)}(0)$ can be expressed by using the Faà di Bruno formula [23, 33, 18]:

$$
f^{(s)}(0)=s ! \sum_{1 \leq|\beta| \leq s} \partial^{\beta} \mathcal{S}(a) \sum_{i=1}^{s} \sum_{P_{i}(s, \beta)} \prod_{j=1}^{i} \frac{\left(\xi_{1}^{\left(\ell_{j}\right)}\right)^{k_{j 1}}}{k_{j 1} !} \frac{\left(\xi_{2}^{\left(\ell_{j}\right)}\right)^{k_{j 2}}}{k_{j 2} !} \frac{\left(\xi_{3}^{\left(\ell_{j}\right)}\right)^{k_{j 3}}}{k_{j 3} !}
$$

for $s>0$ and $f(0)=\mathcal{S}(a)=0$. In (26) the set $P_{i}(s, \beta)$ is given by

$$
\begin{aligned}
P_{i}(s, \beta)=\left\{\left(\ell_{1}, \ldots, \ell_{i}\right),\left(k_{1}, \ldots, k_{i}\right) ; 0<\ell_{1}<\ldots<\ell_{i}\right. & \\
& \left.\left|k_{j}\right|>0, j \in[1, i] ; \quad \sum_{j=1}^{i} k_{j}=\beta, \sum_{j=1}^{i}\left|k_{j}\right| \ell_{j}=s\right\} .
\end{aligned}
$$

From (25)-(26) we infer the relations

$$
\xi^{(s)}(a) \cdot \nabla \mathcal{S}(a)=-\sum_{1<|\beta| \leq s} \partial^{\beta} \mathcal{S}(a) \sum_{i=1}^{s} \sum_{P_{i}(s, \beta)} \prod_{j=1}^{i} \frac{\left(\xi_{1}^{\left(\ell_{j}\right)}\right)^{k_{j 1} 1}}{k_{j 1} !} \frac{\left(\xi_{2}^{\left(\ell_{j}\right)}\right)^{k_{j 2}}}{k_{j 2} !} \frac{\left(\xi_{3}^{\left(\ell_{j}\right)}\right)^{k_{j 3}}}{k_{j 3} !}, \quad \forall q \in \partial \Omega
$$

which play a key role in obtaining recursion relations for the Taylor coefficients $\xi^{(s)}$. Let us already observe that the right-hand side of (28) involves only Taylor coefficients of order less than $s$. This follows from the definition (27) of the multi-index set $P_{i}(s, \beta)$.

Using (28) and the Helmholtz-Hodge decomposition (21)-(23), we shall now show that the determination of the Taylor coefficient $\xi^{(s)}$, knowing all those of lower order, reduces to a vectorvalued non-homogeneous Dirichlet problem and to a non-homogeneous Neumann problem. Let us first note that $\nu$, the normal vector to the boundary $\partial \Omega$, may be expressed in terms of the defining-boundary map $\mathcal{S}$ as

$$
\nu=\frac{\nabla \mathcal{S}}{|\nabla \mathcal{S}|_{2}}
$$

where $|\cdot|_{2}$ is the Euclidean norm and $|\nabla \mathcal{S}|_{2}>0$. Thus the left-hand side of (28) is expressed in terms of the normal component of the Taylor coefficient of order $s$. Upon using (21), we obtain a surface condition involving both the scalar potential $\varphi^{(s)}$ and the vector potential $\Phi^{(s)}$. We choose to impose the vanishing of the vector potential $\Phi^{(s)}$ on $\partial \Omega$; this implies the vanishing of $\nu \cdot \nabla^{\mathrm{L}} \times \Phi^{(s)}$. In this way the equations for the two potential decouple and take the following form. The scalar potential $\varphi^{(s)}$ satisfies the non-homogeneous Neumann boundary value problem

$$
\left\{\begin{array}{lll}
\Delta^{\mathrm{L}} \varphi^{(s)} & =\nabla^{\mathrm{L}} \cdot \xi^{(s)} & \text { in } \Omega \\
\partial_{\nu}^{\mathrm{L}} \varphi^{(s)} & =\xi^{(s)} \cdot \nu & \text { on } \partial \Omega
\end{array}\right.
$$

where it is understood that the right-hand sides of (30) are taken from (20) and (28) $-(29)$, and thus involve only Taylor coefficients $\left\{\xi^{(s)}\right\}_{0 \leq s \leq s-1}$. As to the vector potential $\Phi^{(s)}$, it satisfies the non-homogeneous Dirichlet boundary value problem

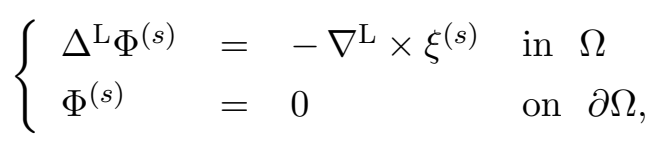


where the right-hand side of (31) is given by (19).

It is straightforward to solve the Helmholtz-Hodge decomposition (21) at the order $s=1$. Indeed, from (19) we obtain that $\nabla^{\mathrm{L}} \times\left(\xi^{(1)}-v_{0}\right)=0$, which implies that there exists a function $f$ such that $\nabla^{\mathrm{L}} f=\xi^{(1)}-v_{0}$. Then, from (20) we obtain that $\nabla^{\mathrm{L}} \cdot \xi^{(1)}=0$, which together with the initial incompressibility condition $\nabla \cdot v_{0}=0$ implies $\Delta^{\mathrm{L}} f=\nabla^{\mathrm{L}} \cdot\left(\xi^{(1)}-v_{0}\right)=0$. Adding the consistent homogeneous Neumann condition $\nu \cdot \nabla^{\mathrm{L}} f=0$ on $\partial \Omega$, to the homogeneous Laplace equation $\Delta^{\mathrm{L}} f=0$ in $\Omega$, we obtain $f=0$ in $\bar{\Omega}$, i.e.

$$
\xi^{(1)}=v_{0} .
$$

This is the starting point of the recursive procedure for the Taylor coefficients.

A few remarks are now in order.

Remark 5 It is standard to prove existence and uniqueness (sometimes up to an additive constant) of the non-homogeneous Dirichlet and Neumann boundary value problems, when the right-hand side and the boundary terms are in suitable functional spaces. Such issues are postponed to Sec. 3.2. where we use such spaces for obtaining a priori estimates.

Remark 6 We have assumed, for convenience that $\Phi^{(s)}=0$ at the boundary. There are other possible choices (see, e.g., [29, 3]), but it is not difficult to show from (19)-(20) and (28) that all lead to the same solution $\xi^{s}$.

Remark 7 We have used a representation of the boundary $\partial \Omega$ by the vanishing of some map $\mathcal{S}$. This is definitely not unique. Furthermore the map $\mathcal{S}$ may be defined either only on the neighborhood of $\partial \Omega$ or more globaly [9, 35]. Will these choices lead to the same time-Taylor coefficients? This can be proved indirectly using Kato's Eulerian classical solution [36], but it would be useful to have a direct Lagrangian proof.

\subsection{Convergence analysis of the formal time series}

Here, we prove that the Lagrangian map $X$ is time-ultradifferentiable in the log-superlinear Faà di Bruno class $\mathcal{C}\{\mathcal{M}\}(]-T, T\left[; \mathscr{C}^{1, \gamma}(\bar{\Omega})\right)$, defined in Sec. 2.1. As a by-product, we obtain the convergence of the time-Taylor expansion (18) of the Lagrangian map $X$, which was explicitly constructed in Sec. 3.1.

Let us introduce the generating function

$$
\zeta(t)=\sum_{s>0}\left\|\xi^{(s)}\right\|_{1, \gamma} M_{s}^{-1} t^{s}
$$

Then, showing that $X \in \mathcal{C}\{\mathcal{M}\}(]-T, T\left[; \mathscr{C}^{1, \gamma}(\bar{\Omega})\right)$ for some $T>0$ is equivalent to proving that $\zeta(t)$ is uniformly bounded on ] $-T, T$, or that the right-hand side of (33) converges uniformly on ]$-T, T[$. To simplify the exposition, we first prove Theorem 2 for initial data that are analytic in the space variable but, nevertheless, as we shall see, we will obtain a priori estimates that depend only on $\left\|v_{0}\right\|_{1, \gamma}$. This is in fact enough for obtaining the general result, since by standard approximation methods, we can regularize $v_{0}$ to render the initial data analytic in space. Then, from results of Refs. [6, 4, 19], we obtain a unique solution that is analytic in time and space for some time. In particular, the displacement $\xi$ is analytic both in time and space and thus the time-Taylor series (33) converges in a non-empty disk. Then, it will be clear from the proof below that the estimates are uniform for the regularized solution. We thus can pass to the limit, keeping the desired a priori estimates by standard arguments, eventually being able to handle initial data $v_{0} \in \mathscr{C}^{1, \gamma}(\bar{\Omega})$. 
Let us now derive the a priori estimates. For this we will use Schauder's regularity estimates in Hölder spaces for the non-homogeneous Neumann and Dirichlet boundary value problems, needed to solve the Helmholtz-Hodge decomposition problems that appear in the previous section. Specifically, for the Dirichlet problem we shall use the Hölder estimates found in Refs. 45, 53, 28, As to the more delicate Neumann problem, there are results using Sobolev spaces in Refs. [45, 49, 28], but for the Hölder case we shall use the recent result of Nardi [54].

We now wish to estimate, in the space $\mathcal{C}^{1, \gamma}$, the Taylor coefficient $\xi^{(s)}$ of order $s$, given by the Helmholtz-Hodge decomposition (21). For this, we need $\mathcal{C}^{2, \gamma}$ estimates on the potentials $\varphi^{(s)}$ and $\Phi^{(s)}$, solutions of the Neumann problem (30) and the Dirichlet problem (31). The aforementioned estimates imply that there exist two constants $C_{\mathrm{D}}=C_{\mathrm{D}}(\Omega, \gamma)$ and $C_{\mathrm{N}}=C_{\mathrm{N}}(\Omega, \gamma)$ such that

$$
\begin{aligned}
\left\|\xi^{(s)}\right\|_{1, \gamma} & \leq\left\|\nabla^{\mathrm{L}} \varphi^{(s)}\right\|_{1, \gamma}+\left\|\nabla^{\mathrm{L}} \times \Phi^{(s)}\right\|_{1, \gamma} \\
& \leq C_{\mathrm{num}}\left(\left\|\varphi^{(s)}\right\|_{2, \gamma}+\left\|\Phi^{(s)}\right\|_{2, \gamma}\right) \\
& \leq C_{\mathrm{DN}}\left(\left\|\nabla^{\mathrm{L}} \cdot \xi^{(s)}\right\|_{0, \gamma}+\left\|\nabla^{\mathrm{L}} \times \xi^{(s)}\right\|_{0, \gamma}+\left\|\xi^{(s)} \cdot \nu\right\|_{1, \gamma}\right),
\end{aligned}
$$

where $C_{\mathrm{DN}}:=C_{\text {num }} \max \left\{C_{\mathrm{D}}, C_{\mathrm{N}}\right\}$ and $C_{\text {num }}$ is a pure numerical constant. Let us note that the operators $\partial_{i}^{\mathrm{L}} \partial_{j}^{\mathrm{L}} \Delta_{\mathrm{L}}^{-1}$ and $\varepsilon_{i k \ell} \partial_{j}^{\mathrm{L}} \partial_{k}^{\mathrm{L}} \Delta_{\mathrm{L}}^{-1}(\cdot)_{\ell}$ are Calderón-Zygmund operators of degre zero, which are continuous endomorphisms in the homogeneous Hölder space $\mathcal{C}^{0, \gamma}(\Omega)$, with a $\gamma$-depending continuity constant varying as $1 / \gamma$ (see, e.g., Chapter 4 of [51, or Chapter 4 of [28]). Therefore, the constants $C_{\mathrm{D}}$ and $C_{\mathrm{N}}$, and consequently $C_{\mathrm{DN}}$ vary in $\gamma$ as $1 / \gamma$. We then must estimate the right-hand side of (34). Using the algebra property (5) and the superlinearity property (3), we find that the divergence of $\xi^{(s)}$, given by the right-hand side of (20), has the following bound

$$
\frac{\left\|\nabla^{\mathrm{L}} \cdot \xi^{(s)}\right\|_{0, \gamma}}{M_{s}} \leq 6 C_{a} M_{0} \sum_{0<m<s} \frac{\left\|\xi^{(m)}\right\|_{1, \gamma}}{M_{m}} \frac{\left\|\xi^{(s-m)}\right\|_{1, \gamma}}{M_{s-m}}+6 C_{a}^{2} M_{0}^{2} \sum_{l+m+n=s} \frac{\left\|\xi^{(l)}\right\|_{1, \gamma}}{M_{l}} \frac{\left\|\xi^{(m)}\right\|_{1, \gamma}}{M_{m}} \frac{\left\|\xi^{(n)}\right\|_{1, \gamma}}{M_{n}}
$$

where $C_{a}$ is appearing in the algebra bound (5). It follows that

$$
\sum_{s>0}\left\|\nabla^{\mathrm{L}} \cdot \xi^{(s)}\right\|_{0, \gamma} M_{s}^{-1} t^{s} \leq 6 C_{a} M_{0}\left(\zeta^{2}+C_{a} M_{0} \zeta^{3}\right)
$$

In an exactly similar way we can bound the curl of $\xi^{(s)}$, given by the right-hand side of (19):

$$
\frac{\left\|\nabla^{\mathrm{L}} \times \xi^{(s)}\right\|_{0, \gamma}}{M_{s}} \leq \frac{1}{M_{1}}\left\|\omega_{0}\right\|_{0, \gamma} \delta_{1 s}+\frac{3}{2} C_{a} M_{0} \sum_{0<m<s} \frac{\left\|\xi^{(m)}\right\|_{1, \gamma}}{M_{m}} \frac{\left\|\xi^{(s-m)}\right\|_{1, \gamma}}{M_{s-m}}
$$

so that

$$
\sum_{s>0}\left\|\nabla^{\mathrm{L}} \times \xi^{(s)}\right\|_{0, \gamma} M_{s}^{-1} t^{s} \leq \frac{1}{M_{1}}\left\|\omega_{0}\right\|_{0, \gamma} t+\frac{3}{2} C_{a} M_{0} \zeta^{2} .
$$

Multiplying (34) by $M_{s}^{-1} t^{s}$, summing the result over the index $s$, and using (35)-(36), we then obtain

$$
\zeta(t) \leq C_{\mathrm{DN}}\left(\frac{15}{2} C_{a} M_{0} \zeta^{2}(t)+6 C_{a}^{2} M_{0}^{2} \zeta^{3}(t)+\frac{1}{M_{1}}\left\|\omega_{0}\right\|_{0, \gamma} t+\sum_{s>0}\left\|\xi^{(s)} \cdot \nu\right\|_{1, \gamma} M_{s}^{-1} t^{s}\right) .
$$


It remains to control the boundary term in the left-hand side of (37). Since we assumed $\mathcal{S} \in$ $\mathcal{C}\{\mathcal{M}\}(\partial \Omega ; \mathbb{R})$, there exist two constants $C_{\mathcal{S}}$ and $R_{\mathcal{S}}$ such that

$$
\left\|\partial^{\beta} \mathcal{S}\right\|_{L^{\infty}} \leq C_{\mathcal{S}} R_{\mathcal{S}}^{-|\beta|}|\beta| ! M_{|\beta|}
$$

Let us define

$$
C_{\nabla \mathcal{S}}:=\left\||\nabla \mathcal{S}(q)|_{2}^{-1}\right\|_{1, \gamma} .
$$

Then, using (28) $-(29)$, (15) and (38), we obtain

$$
\begin{aligned}
\frac{\left\|\xi^{(s)} \cdot \nu\right\|_{1, \gamma}}{M_{s}} \leq & C_{a} C_{\nabla \mathcal{S}}\left\|\xi^{(s)} \cdot \nabla \mathcal{S}\right\|_{1, \gamma} M_{s}^{-1} \\
\leq & C_{\nabla \mathcal{S}} \sum_{1 \leq|\beta| \leq s}\left(\frac{C_{a}}{R_{\mathcal{S}}}\right)^{|\beta|+1} \frac{C_{\mathcal{S}}}{R_{\mathcal{S}}}(|\beta|+2) ! \frac{M_{|\beta|+2}}{M_{s}} \sum_{i=1}^{s} \sum_{P_{i}(s, \beta)} \prod_{j=1}^{i} \frac{\left\|\xi^{\left(\ell_{j}\right)}\right\|_{1, \gamma}^{\left|k_{j}\right|}}{k_{j} !} \\
\leq & C_{\nabla \mathcal{S}} \frac{C_{\mathcal{S}}}{R_{\mathcal{S}}} \sum_{1 \leq|\beta| \leq s}\left(\frac{C_{a}}{R_{\mathcal{S}}}\right)^{|\beta|+1}(|\beta|+2)(|\beta|+1)|\beta| ! \frac{M_{|\beta|+2}}{M_{|\beta|+1}} \frac{M_{|\beta|+1}}{M_{|\beta|}} \\
& \sum_{i=1}^{s} \sum_{P_{i}(s, \beta)} \frac{M_{|\beta|} M_{\ell_{1}}^{\left|k_{1}\right|} \ldots M_{\ell_{i}}^{\left|k_{i}\right|}}{M_{s}} \prod_{j=1}^{i}\left(\frac{\left\|\xi^{\left(\ell_{j}\right)}\right\|_{1, \gamma}}{M_{\ell_{j}}}\right)^{\left|k_{j}\right|} \frac{1}{k_{j} !} .
\end{aligned}
$$

It is now convenient to use the notation

$$
\alpha_{1}:=\ell_{1}, \ldots, \quad \alpha_{\left|k_{1}\right|}:=\ell_{1}, \quad \alpha_{\left|k_{1}\right|+1}:=\ell_{2}, \ldots, \quad \alpha_{\left|k_{1}\right|+\left|k_{2}\right|}:=\ell_{2}, \ldots, \quad \alpha_{\left|k_{1}\right|+\ldots+\left|k_{i}\right|}:=\ell_{i},
$$

in terms of which we have

$$
\begin{aligned}
M_{\ell_{1}}^{\left|k_{1}\right|} \ldots M_{\ell_{i}}^{\left|k_{i}\right|} & =M_{\alpha_{1}} \ldots M_{\alpha_{\left|k_{1}\right|}} M_{\alpha_{\left|k_{1}\right|+1}} \ldots M_{\alpha_{\left|k_{1}\right|+\left|k_{2}\right|}} \ldots M_{\alpha_{\left|k_{1}\right|+\ldots+\left|k_{i}\right|}} \\
& =M_{\alpha_{1}} \ldots M_{\alpha_{|\beta|} \mid} .
\end{aligned}
$$

Using (40) and (33), equation (39) becomes

$$
\begin{aligned}
\frac{\left\|\xi^{(s)} \cdot \nu\right\|_{1, \gamma}}{M_{s}} \leq C_{a} \frac{C_{\mathcal{S}}}{R_{\mathcal{S}}} \frac{C_{\nabla \mathcal{S}}}{R_{\mathcal{S}}} \sum_{1 \leq|\beta| \leq s}(|\beta|+2)(|\beta|+1)\left(\frac{C_{a}}{R_{\mathcal{S}}}\right)^{|\beta|}|\beta| ! \frac{M_{|\beta|+2}}{M_{|\beta|+1}} \frac{M_{|\beta|+1}}{M_{|\beta|}} \\
\qquad \sum_{i=1}^{s} \sum_{P_{i}(s, \beta)} \frac{M_{|\beta|} M_{\alpha_{1}} \ldots M_{\alpha_{|\beta|}}}{M_{s}} \prod_{j=1}^{i} \frac{\left(\partial^{\ell_{j}} \zeta(0)\right)^{\left|k_{j}\right|}}{k_{j} !\left(\ell_{j} !\right)^{\left|k_{j}\right|}} .
\end{aligned}
$$

On the one hand, the differentiability property (2) leads to

$$
\frac{M_{|\beta|+2}}{M_{|\beta|+1}} \frac{M_{|\beta|+1}}{M_{|\beta|}} \leq C_{\mathrm{d}}^{2|\beta|+1} .
$$

On the other hand, the FdB-stability property (3) leads to

$$
\frac{M_{|\beta|} M_{\alpha_{1}} \ldots M_{\alpha_{|\beta|}}}{M_{s}} \leq \frac{M_{\alpha_{1}+\ldots+\alpha_{|\beta|}}}{M_{s}} \leq 1 .
$$


From (42)-(43), estimate (41) becomes

$$
\begin{aligned}
\frac{\left\|\xi^{(s)} \cdot \nu\right\|_{1, \gamma}}{M_{s}} \leq & C_{a} C_{\mathrm{d}} \frac{C_{\mathcal{S}}}{R_{\mathcal{S}}} \frac{C_{\nabla \mathcal{S}}}{R_{\mathcal{S}}} \frac{(s+2)(s+1)}{s !} \\
& s ! \sum_{1 \leq|\beta| \leq s}\left(\frac{C_{a} C_{\mathrm{d}}}{R_{\mathcal{S}}}\right)^{|\beta|}|\beta| ! \sum_{i=1}^{s} \sum_{P_{i}(s, \beta)} \prod_{j=1}^{i} \frac{\left(\partial^{\ell_{j}} \zeta(0)\right)^{\left|k_{j}\right|}}{k_{j} !\left(\ell_{j} !\right)^{\left|k_{j}\right|}} \\
\leq & C_{a} C_{\mathrm{d}}^{2} \frac{C_{\mathcal{S}}}{R_{\mathcal{S}}} \frac{C_{\nabla \mathcal{S}}}{R_{\mathcal{S}}} \frac{(s+2)(s+1)}{s !} \\
& s ! \sum_{1 \leq|\beta| \leq s}\left(\partial^{\beta} G\right)(0,0,0) \sum_{i=1}^{s} \sum_{P_{i}(s, \beta)} \prod_{j=1}^{i} \frac{\left(\partial^{\ell_{j}} \zeta(0)\right)^{\left|k_{j}\right|}}{k_{j} !\left(\ell_{j} !\right)^{\left|k_{j}\right|}}
\end{aligned}
$$

where the map $G: \mathbb{R}^{3} \rightarrow \mathbb{R}$ is defined by

$$
G\left(x_{1}, x_{2}, x_{3}\right)=\prod_{i=1}^{3}\left(1-\frac{C_{a} C_{\mathrm{d}}^{2}}{R_{\mathcal{S}}} x_{i}\right)^{-1 / 3},
$$

and satisfies

$$
\left(\partial^{\beta} G\right)(0,0,0)=\partial_{z}^{|\beta|} G(z, z, z)_{\mid z=0}=|\beta| !\left(\frac{C_{a} C_{\mathrm{d}}^{2}}{R_{\mathcal{S}}}\right)^{|\beta|}
$$

Setting

$$
\begin{gathered}
g^{(s)}(0):=s ! \sum_{1 \leq|\beta| \leq s}\left(\partial^{\beta} G\right)(0,0,0) \sum_{i=1}^{s} \sum_{P_{i}(s, \beta)} \prod_{j=1}^{i} \frac{\left(\partial^{\ell_{j}} \zeta(0)\right)^{\left|k_{j}\right|}}{k_{j} !\left(\ell_{j} !\right)^{\left|k_{j}\right|}} \\
C_{\mathrm{d} a \mathcal{S}}:=C_{a} C_{\mathrm{d}}^{2} \frac{C_{\mathcal{S}}}{R_{\mathcal{S}}} \frac{C_{\nabla \mathcal{S}}}{R_{\mathcal{S}}}, \quad C_{\mathcal{S} a \mathrm{~d}}^{-1}:=\frac{C_{a} C_{\mathrm{d}}^{2}}{R_{\mathcal{S}}}
\end{gathered}
$$

and using the Faà di Bruno formula, we can rewrite (44) as

$$
\begin{aligned}
\sum_{s>0}\left\|\xi^{(s)} \cdot \nu\right\|_{1, \gamma} M_{s}^{-1} t^{s} & \leq C_{\mathrm{d} a \mathcal{S}} \sum_{s>0}(s+2)(s+1) \frac{g^{(s)}(0)}{s !} t^{s} \\
& \leq C_{\mathrm{d} a \mathcal{S}} \frac{d^{2}}{d t^{2}}\left(t^{2} g(t)\right) \\
& \leq C_{\mathrm{d} a \mathcal{S}} \frac{d^{2}}{d t^{2}}\left(t^{2} G(\zeta(t), \zeta(t), \zeta(t))\right) \\
& \leq C_{\mathrm{d} a \mathcal{S}} \frac{d^{2}}{d t^{2}}\left(\frac{t^{2}}{1-C_{\mathcal{S} a \mathrm{~d}}^{-1} \zeta(t)}\right) .
\end{aligned}
$$

Combining (37) and (46), we obtain the final a priori estimate

$$
\zeta(t) \leq C_{\mathrm{DN}}\left(\frac{15}{2} C_{a} M_{0} \zeta^{2}(t)+6 C_{a}^{2} M_{0}^{2} \zeta^{3}(t)+\frac{1}{M_{1}}\left\|\omega_{0}\right\|_{0, \gamma} t+C_{\mathrm{d} a \mathcal{S}} \frac{d^{2}}{d t^{2}}\left(\frac{t^{2}}{1-C_{\mathcal{S} a \mathrm{~d}}^{-1} \zeta(t)}\right)\right)
$$

which we rewrite as

$$
\frac{d^{2}}{d t^{2}}\left(\frac{t^{2}}{C_{\mathcal{S} a \mathrm{~d}}^{-1} \zeta(t)-1}\right) \leq h(t)
$$


where

$$
\begin{aligned}
h(t) & :=C_{\mathrm{d} a \mathcal{S}}^{-1}\left(6 C_{a}^{2} M_{0}^{2} \zeta^{3}(t)+\frac{15}{2} C_{a} M_{0} \zeta^{2}(t)-C_{\mathrm{DN}}^{-1} \zeta(t)+\frac{1}{M_{1}}\left\|\omega_{0}\right\|_{0, \gamma} t\right) \\
& =\frac{d^{2}}{d t^{2}}\left(\int_{0}^{t}(t-\tau) h(\tau) d \tau\right) .
\end{aligned}
$$

Twice integrating the differential inequality (47), and assuming that $1-C_{\mathcal{S} a \mathrm{~d}}^{-1} \zeta(t) \geq 0$, for the time being, we obtain

$$
-1 \leq\left(1-C_{\mathcal{S} a \mathrm{~d}}^{-1} \zeta(t)\right) \frac{1}{t^{2}} \int_{0}^{t} h(\tau) d \tau .
$$

A sufficient condition for this differential inequality to hold is to have simultaneously

$$
1-C_{\mathcal{S} a \mathrm{~d}}^{-1} \zeta(t) \geq 0, \quad \text { and } \quad Q(\zeta):=6 C_{a}^{2} M_{0}^{2} \zeta^{3}(t)+\frac{15}{2} C_{a} M_{0} \zeta^{2}(t)-C_{\mathrm{DN}}^{-1} \zeta(t)+\Gamma(t) \geq 0,
$$

where we have set $\Gamma(t):=\left(\left\|\omega_{0}\right\|_{0, \gamma} / M_{1}\right) t$. The discriminant of the cubic polynomial $Q(\zeta)$,

$$
\Delta:=\frac{1}{54 C_{a}^{6} M_{0}^{6}}\left(\frac{25}{8}+\frac{1}{C_{\mathrm{DN}}}\right)^{3}-\frac{9}{C_{a}^{4} M_{0}^{4}}\left(\frac{5}{72} \frac{1}{C_{a} M_{0}}\left(\frac{25}{12}+\frac{1}{C_{\mathrm{DN}}}\right)+\frac{\Gamma}{6}\right)^{2},
$$

is positive at small times, when $\Gamma$ is sufficiently small, whereby $Q(\zeta)$ has three real roots $\zeta_{i}$. The polynomial has two local extrema at points of different signs, hence it has roots of both signs. Since by Viète's theorem the product of the roots is negative, two roots are positive and one is negative. The second inequality of (48) implies

$$
\zeta \leq \zeta_{2}(\Gamma)
$$

where $\zeta_{2}$ is the intermediate root, i.e. the smaller of the two positive roots.

We determine now the largest time $t_{c}$, for which bound (49) holds. Differentiating the equation for the roots of polynomial (48) with respect to $\Gamma$, we find

$$
\frac{\partial \zeta_{i}}{\partial \Gamma}=-\left(\left.\frac{\partial Q}{\partial \zeta}\right|_{\zeta=\zeta_{i}}\right)^{-1}
$$

Consequently, on increasing $\Gamma$ from zero, the root $\zeta_{2}$ monotonically increases from zero and $\zeta_{3}$ monotonically decreases till the two roots collide (i.e. $\zeta_{2}=\zeta_{3}$ ) at the critical value $\Gamma=\Gamma_{c}$, determined by the equation $\Delta=0$. This corresponds to the critical time $t_{c}=\Gamma_{c} M_{1} /\left\|\omega_{0}\right\|_{0, \gamma}$. Setting $t_{\mathcal{S} a \mathrm{~d}}=Q\left(C_{\mathcal{S} a \mathrm{~d}}\right) M_{1} /\left\|\omega_{0}\right\|_{0, \gamma}$, an upper bound of the radius of convergence $T$ of the generating function $\zeta$ is given by the smaller of $t_{\mathcal{S} a \mathrm{~d}}$ and $t_{c}$. Therefore, we obtain

$$
\left.\zeta(t) \leq \min \left\{C_{\mathcal{S} a \mathrm{~d}}, \zeta_{2}\left(\Gamma_{c}\right)\right\}, \quad t \in\right]-T, T\left[, \quad T=\min \left\{t_{\mathcal{S} a \mathrm{~d}}, t_{c}\right\},\right.
$$

and the sufficient condition (48) is indeed satisfied. We observe, from the definition of $t_{\mathcal{S} a \mathrm{~d}}$ and $t_{c}$ that the maximum time for which the Lagrangian map is time-ultradifferentiable is controlled by the Hölder continuity of the initial vorticity $\left\|\omega_{0}\right\|_{0, \gamma}$ and the "radius of ultradifferentiability" $R_{\mathcal{S}}$ of the boundary.

Finally, we note that our solution was constructed from a Taylor expansion around $t=0$. However, we can restart the process from any time $t_{*}>0$, at which the vorticity has $\mathcal{C}^{0, \eta}$-regularity for some $\eta>0$ (which may be less than $\gamma$ ). Then, ultradifferentiability in time persists on the interval $\left[t_{*}, t_{*}+T_{*}\right.$ [ for some positive $T_{*}$. This process can be continued until the vorticity ceases to have any $\mathcal{C}^{0, \eta}$-regularity. Clearly the Lagrangian map remains time-ultradifferentiable as long as the vorticity is Hölder continuous. This completes our proof. 


\section{Concluding remarks}

For 3D incompressible Euler flow with an initial vorticity that is Hölder continuous, we have extended the results of Refs. [25, 67] on time-analyticity of the Lagrangian map to the case of wall-bounded flow with a fixed impermeable boundary that is analytic in its shape. We have also obtained similar regularity results when the boundary is in a suitable class of ultradifferentiability.

An important feature that singles out our proof is that it is constructive, in the sense that it leads to explicit recursion relations for the time-Taylor coefficients of the Lagrangian map. This feature was crucial in allowing the development of an efficient Cauchy-Lagrangian numerical scheme for the case without boundary [56. Such a scheme can in principle be carrried over to the case with a boundary; this will, of course, require the numerical solution of the elliptic (Poisson) equations with both Dirichlet and Neumann boundary conditions involved in the Helmholtz-Hodge decompositions. For the case of a boundary that is not analytic, but in a suitable ultradifferential class, the time-Taylor series will generally be divergent for any $t>0$, so that some resummation method (e.g., by a Borel transformation [55]) must also be used for the numerical implementation.

Once a variant of the Cauchy-Lagrangian scheme, adapted to solid boundaries has been developped, it will be of interest to revisit the issue of finite time blow-up via state-of-the-art simulations.

So far, we have only considered the case of flow within a bounded simply connected domain. The extension of our results to outer flow (e.g. flow in an infinite domain around a bounded obstacle) and to multiply connected domains will be considered later.

It is also of interest to handle the extension of our results to other flow equations. One obvious case is the Euler-Poisson equation for which a Lagrangian time-analyticity result was obtained [67, 59]. This was done in the absence of boundary and in a cosmological context of attractive gravitational forces. Boundaries are usually not considered in cosmology. However, they play an important part in plasma physics because, for example, fusion facilities are always enclosed. Of particular interest for plasmas is the Euler-Poisson equation with a repulsive electrostatic force, which will be considered in subsequent work.

\section{Acknowledgements}

We are grateful to Claude Bardos and László Székelyhidi for many fruitful discussions. This work was supported by the VLASIX and EUROFUSION projects respectively under the grants No ANR-13-MONU-0003-01 and EURATOM-WP15-ENR-01/IPP-01. We have also benefitted from our participation in the Wolfgang Pauli Institute workshop "Euler and Navier-Stokes Equations and Connected Topics," Vienna, December 14-18, 2015.

\section{References}

[1] R.A. Adams, Sobolev spaces, Academic Press, 1975.

[2] V.I. Arnold, Sur la géometrie différentielle des groupes de Lie de dimension infinie et ses applications à l'hydrodynamique des fluides parfaits, Ann. Inst. Fourier 16 (1966) 319-361.

[3] C. Amrouche, C. Bernardi, M. Dauge, V. Girault, Vector potentials in three-dimensional nonsmooth domains, Math. Meth. Appl. Sci. 21 (1998) 823-864.

[4] C. Bardos, S. Benachour, Domaine d'analyticité des solutions de l'équation d'Euler dans un ouvert de $\mathbb{R}^{n}$, Ann. Scuola Norm. Sup. Pisa Cl. Sci. $4^{e}$ série 4 (1977) 647-687.

[5] C. Bardos, E. Titi, Loss of smoothness and energy conserving rough weak solutions for the 3d Euler equations, Dis. Cont. Dyn. Syst. S 3 (2010) 185-197. 
[6] S. Benachour, Analyticité des solutions périodiques de l'équation d'Euler en trois dimensions, C. R. Acad. Sci. Paris A 283 (1976) 107-110.

[7] F. Bernardeau, S. Colombi, E. Gaztañaga, R. Scoccimarro Large-scale structure of the universe and cosmological perturbation theory, Phys. Rep. 367 (2002) 1-248.

[8] A. Beurling, Quasi-analyticity and general distributions, Lecture Notes, AMS Summer Institute, Stanford, 1961.

[9] J.-P. Bourguignon, H. Brézis, Remarks on the Euler equation, J. Functional Analysis 15 (1974) 341-363.

[10] T. Buckmaster, C. De Lellis, P. Isett, L. Székelyhidi, Anomalous dissipation for 1/5-Hölder Euler flows Ann. of Math. 182 (2015) 127-172.

[11] T. Carleman, Sur les fonctions quasi-analytiques, Gauthiers Villars, Paris, 1926.

[12] H. Cartan, Théorie élémentaire des fonctions analytiques d'une ou plusieurs variables complexes, Hermann, Paris, 1961.

[13] A.L. Cauchy. Sur l'état du fluide à une époque quelconque du mouvement. Mémoires extraits des recueils de l'Académie des sciences de l'Institut de France, Théorie de la propagation des ondes à la surface d'un fluide pesant d'une profondeur indéfinie (Extraits des Mémoires présentés par divers savants à l'Académie royale des Sciences de l'Institut de France et imprimés par son ordre. Sciences mathématiques et physiques. Tome I, 1827 Seconde Partie. pp. 33-73 (1827).

[14] J.-Y. Chemin, Régularité de la trajectoire des particules d'un fluide parfait incompressible remplissant l'espace, J. Math. Pures Appl. 71 (1992) 407-417.

[15] J.-Y. Chemin, Fluides parfaits incompressibles, Astérisque 230 1995. (Engl. transl.: J.-Y. Chemin, Perfect Incompressible Fluids, Clarendon press, Oxford, 1998.)

[16] P. Constantin, V. Vicol, J. Wu, Analyticity of Lagrangian trajectories for well-posed inviscid incompressible fluid models, Adv. Math. 285 (2015) 352-393.

[17] P. Constantin, I. Kukavica, V. Vicol, Contrast between Lagrangian and Eulerian analytic regularity properties of Euler equations, Ann. I. H. Poincaré - AN (2015) doi:10.1016/j.anihpc.2015.07.002.

[18] G.M. Constantine, T.H. Savits, A multivariate Faà di Bruno formula with applications, Trans. Amer. Math. Soc. 348 (1996) 503-520.

[19] J.-M. Delort, Estimations fines pour des opérateurs pseudo-différentiels analytiques sur un ouvert à bord de $\mathbb{R}^{n}$, application aux équations d'Euler Comm. Part. Diff. Eq. 10 (1985) 1465-1525.

[20] A. Denjoy, Sur les fonctions quasi-analytiques de variable réelle, C. R. Acad. Sci. Paris A 123 (1921) $1320-1322$.

[21] A. Dutrifoy, Precise regularity results for the Euler equations, J. Math. Anal. Appl. 282 (2003) 177-200.

[22] D.G. Ebin, J.E. Marsden, Groups of diffeomorphisms and the motion of an incompressible fluid, Ann. Math. 92 (1970) 102-163.

[23] C.F. Faà di Bruno, Note sur une nouvelle formule du calcul différentiel, Quart. J. Math. 1 (1855) 359-360.

[24] C. Foias, R. Teman, Remarques sur les équations de Navier-Stokes stationnaires et les phénomènes successifs de bifurcation, Ann. Scuola Norm. Sup. Pisa Cl. Sci. $4^{e}$ série 5 (1978) 29-63.

[25] U. Frisch, V. Zheligovsky, A very smooth ride in rough sea, Commun. Math. Phys. 326 (2014) 499-505.

[26] U. Frisch, B. Villone, Cauchy's almost forgotten Lagrangian formulation of the Euler equation for 3D incompressible flow, Eur. Phys. J. H. 39 (2014) 325-351. 
[27] P. Gamblin, Système d'Euler incompressible et régularité microlocale analytique, Ann. Inst. Fourier 44 (1994) 1449-1475.

[28] D. Gilbarg, N.S. Trudinger, Elliptic partial differential equationsof second order, Springer-Verlag, 1998.

[29] V. Girault, P.-A. Raviart, Finite element methods for Navier-Stokes equations, theory and algorithms, Springer-Verlag, 1986.

[30] O. Glass, F. Sueur, T. Takahashi, Smoothness of the motion of a rigid body immersed in an incompressible perfect fluid, Ann. Scient. Éc. Norm. Sup. $4^{e}$ série 45 (2012) 1-51.

[31] N.M. Gyunter [Günther], Über ein Hauptproblem der Hydrodynamik [On a main problem of hydrodynamics], Math. Z. 24 (1926) 448-499.

[32] N.M. Gyunter [Gunther], La théorie du potentiel et ses applications aux problèmes fondamentaux de la physique mathématique, Gauthier-Villars, Paris, 1934. (Engl. transl.: N.M. Gyunter [Günter], Potential theory, and its applications to basic problems of mathematical physics, Frederick Ungar Publ., NY, 1967.)

[33] H. Gzyl, Multidimensional extension of Faà di Bruno's formula, J. Math. Anal. Appl. 116 (1986) $450-455$.

[34] L. Hörmander, The analysis of linear partial differential operators. I, Springer-Verlag, 1983.

[35] T. Kato, On the smoothness of trajectories in incompressible perfect fluids, Contemp. Math. 263 (2000) 109-130.

[36] T. Kato, Two manuscripts left by late Professor Tosio Kato in his personal computer, in Tosio Kato's method and principle for evolution equations in mathematical physics 2001, 260-274.

[37] H. Komatsu, Ultradistributions. I. Structure theorems and a characterization, J. Fac. Sci. Univ. Tokyo Sect. IA Math. 20 (1973) 25-105.

[38] S.G. Krantz, H.R.. Parks, A primer of real analytic functions. Birkhaüser, 2002.

[39] A. Kriegl, P.W. Michor, A. Rainer, The convenient setting for non-quasianalytic Denjoy-Carleman differentiable mappings. J. Funct. Anal. 256 (2009) 3510-3544.

[40] A. Kriegl, P.W. Michor, A. Rainer, The convenient setting for quasianalytic Denjoy-Carleman differentiable mappings. J. Funct. Anal. 261 (2011) 1799-1834.

[41] A. Kriegl, P.W. Michor, A. Rainer, The convenient setting for Denjoy-Carleman differentiable mappings of Beurling and Roumieu type. Rev. Mat. Complut. 28 (2015) 549-597.

[42] I. Kukavica, V. Vicol, On the radius of analyticity of solutions to the three-dimensional Euler equations, Proc. Am. Math. Soc. 137 (2009) 669-677.

[43] I. Kukavica, V. Vicol, On the analyticity and Gevrey-class regularity up to the boundary for the Euler equations, Nonlinearity 24 (2011) 765-796.

[44] I. Kukavica, V. Vicol, The domain of analyticity of solutions to the three-dimensional Euler equations in a half-plane, Dis. Cont. Dyn. Syst. A 29 (2011) 285-303.

[45] O. Ladyzhenskaya, N. Uraltseva, Linear and quasilinear elliptic equations. Academic Press, 1968.

[46] D. Levermore, M. Oliver, Analyticity of solutions for a generalized Euler equation. J. Differential Equations 133 (1997) 321-339.

[47] L. Lichtenstein, Über einige Existenzprobleme der Hydrodynamik [On some existence problems of hydrodynamics], Math. Z. 23 (1927) 196-323

[48] L. Lichtenstein, Grundlagen der Hydromechanik [Foundations of Hydromechanics], Springer, 1929. 
[49] J.-L. Lions, E. Magenes, Non-homogeneous boundary value problems and applications, Vol I, SpringerVerlag, 1972.

[50] J.-L. Lions, E. Magenes, Non-homogeneous boundary value problems and applications, Vol III, SpringerVerlag, 1972.

[51] A. Majda, E. Bertozzi, Vorticity and incompressible flow, Cambridge University Press, 2002.

[52] S. Mandelbrojt, Analytic functions and classes of infinitely differentiable functions, Lecture Notes at the Rice Institute, 1942.

[53] C. Miranda, Partial differential equations of elliptic type, Springer-Verlag, 1970.

[54] G. Nardi, Schauder estimate for solutions of Poisson's equation with Neumann boundary condition, L'enseignement Mathématique 60 (2014) 421-435.

[55] W. Pauls, U. Frisch, A Borel transform method for locating singularities of Taylor and Fourier series, J. Stat. Phys. 127 (2007) 1095-1119.

[56] O. Podvigina, V. Zheligovsky, U. Frisch, The Cauchy-Lagrangian method for numerical analysis of Euler flow, J. Comput. Phys. 306 (2016) 320-342.

[57] A. Rainer, G. Schindl, Composition in ultradifferentiable classes, Stud. Math. 224 (2014) 97-131.

[58] A. Rainer, G. Schindl, Equivalence of stability properties for ultradifferentiable function classes, Rev. R. Acad. Cienc. Exactas Fis. Nat. Ser. A Math. (2016).

[59] C. Rampf, B. Villone, U. Frisch, How smooth are particle trajectories in a $\Lambda$ CDM Universe?, MNRAS 452 (2015) 1421-1436.

[60] C. Roumieu, Ultra-distributions définies sur $\mathbb{R}^{n}$ et sur certaines classes de variétés différentiables, J. Analyse Math. 10 (1962/1963), 153-192.

[61] W. Rudin, Real and complex analysis, McGraw-Hill, 1987.

[62] P. Serfati, Etude mathématique de flammes infiniment minces en combustion. Résultats de structure et de régularité pour l'équation d'Euler incompressible, Ph.D. Thesis, Université Paris 6, 1992.

[63] P. Serfati, Equation d'Euler et holomorphies à faible régularité spatiale, C. R. Acad. Sci. Paris I 320 (1994) 175-180.

[64] P. Serfati, Structures holomorphes à faible régularité spatiale en mécanique des fluides, J. Math. Pures Appl. 74 (1995) 95-104.

[65] A. Shnirelman, On the analyticity of particle trajectories in the ideal incompressible fluid. arXiv preprint arXiv:1205.5837, 2012.

[66] V. Thilliez, On quasianalytic local rings, Expo. Math. 26 (2008) 1-23.

[67] V. Zheligovsky, U. Frisch, Time-analyticity of Lagrangian particle trajectories in ideal fluid flow, J. Fluid Mech. 749 (2014) 404-430.

[68] V. Zheligovsky, A priori bounds for Gevrey-Sobolev norms of space-periodic three-dimensional solutions to equations of hydrodynamic type, Adv. Differential Equations 16 (2011) 955-976. 